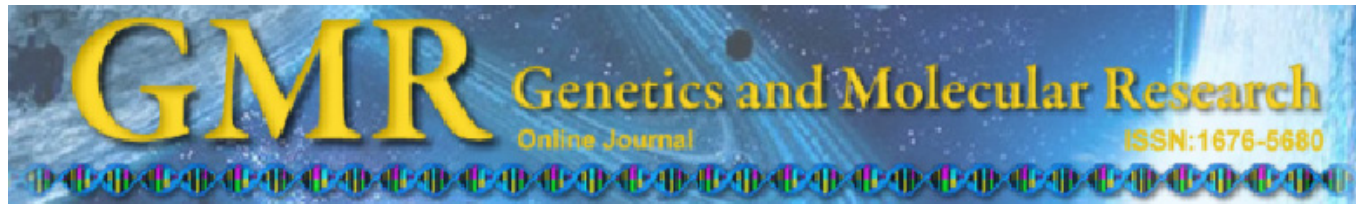

Short Communication

\title{
Characterization of Trypanosoma brucei gambiense variant surface glycoprotein LiTat 1.5
}

\author{
L. Van Nieuwenhove ${ }^{1 *}$, S. Rogé ${ }^{1 *}$, V. Lejon ${ }^{1}, Y_{.}$Guisez $^{2}$ and P. Büscher $^{1}$ \\ ${ }^{1}$ Department of Biomedical Sciences, Institute of Tropical Medicine, \\ Antwerp, Belgium \\ ${ }^{2}$ Department of Biology, University of Antwerp, \\ Antwerp, Belgium \\ *These authors contributed equally to this study. \\ Corresponding author: P. Büscher \\ E-mail: pbuscher@itg.be
}

Genet. Mol. Res. 11 (2): 1260-1265 (2012)

Received September 8, 2011

Accepted January 9, 2012

Published May 9, 2012

DOI http://dx.doi.org/10.4238/2012.May.9.5

\begin{abstract}
At present, all available diagnostic antibody detection tests for Trypanosoma brucei gambiense human African trypanosomiasis are based on predominant variant surface glycoproteins (VSGs), such as VSG LiTat 1.5. During investigations aiming at replacement of the native VSGs by recombinant proteins or synthetic peptides, the sequence of VSG LiTat 1.5 was derived from cDNA and direct $\mathrm{N}$-terminal amino acid sequencing. Characterization of the VSG based on cysteine distribution in the amino acid sequence revealed an unusual cysteine pattern identical to that of VSG Kinu 1 of $T . b$. brucei. Even though both VSGs lack the third of four conserved cysteines typical for type A N-terminal domains, they can be classified as type A.
\end{abstract}

Key words: Trypanosoma brucei gambiense; VSG; LiTat 1.5; Amino acid sequence; Cysteine pattern 


\section{INTRODUCTION}

In mammalian hosts, the entire cell surface of African trypanosomes, including the flagellum, is covered by a dense monolayer of variant surface glycoproteins (VSGs). This protective coat has several functions, e.g.: 1) it shields the invariant antigens from the immune system, 2) antigenic variation through frequent stochastic switching of VSG permits trypanosomes to circumvent the specific antibody response that was raised against earlier variable antigen types (VAT). The trypanosome genome contains $>1000$ VSG genes, but only one is expressed at a time (Berriman et al., 2005). The VSG dimers are tethered to the cell surface by two glycosylphosphatidylinositol anchors that may allow high packing density and that play a role in correct transportation and recycling of the VSG (Ferguson et al., 1988a,b; Engstler et al., 2004, 2007). Each VSG monomer contains at least one N-linked oligosaccharide and consists of two domains that are separated by a hinge region. On the living trypanosome, the hyper-variable N-terminal domain (350-400 residues) is exposed to the immune system while the relatively conserved C-terminal domain (40-80 residues) is hidden from it by the intact VSG coat. VSGs are classified based on 1) the position of cysteines in the mature protein sequence, 2) conserved $\mathrm{N}$-linked glycosylation sites, 3 ) the $\mathrm{C}$-terminal residue, and 4) the C-terminal hydrophobic GPI-anchor signal. The N-terminal domain is classified into three types, A, B and C (Carrington et al., 1991), although type C has been proposed to be classified as type A (Marcello and Barry, 2007). Despite their high primary sequence heterogeneity, all N-terminal domains fold with a similar tertiary structure, necessary to form the densely packed protective VSG coat, which exposes only a limited subset of immunogenic epitopes (Freymann et al., 1984; Blum et al., 1993; Chattopadhyay et al., 2005; Schwede and Carrington, 2010; Schwede et al., 2011). There are six types of C-terminal domains; types 2, 4 and 5 are single domains, while types 1, 3 and 6 are di-domains (Carrington et al., 1991; Marcello and Barry, 2007; Schwede and Carrington, 2010). The type 2 domain of MITat 1.2 and the type 1 domain of ILTat 1.24 share the same tertiary structure, arguing for conservation of the tertiary structure of both $\mathrm{N}$ - and C-terminal domains (Schwede and Carrington, 2010). All combinations of N-terminal with C-terminal domains seem to be possible (Hutchinson et al., 2003).

One of the predominant VSGs of Trypanosoma brucei gambiense, causing the chronic form of sleeping sickness or human African trypanosomiasis (HAT), is VSG LiTat 1.5 (Van Meirvenne et al., 1995). All currently available antibody detection tests for gambiense HAT, i.e., card agglutination test for trypanosomiasis (CATT) (Magnus et al., 1978), LATEX/T. $b$. gambiense and ELISA/T. b. gambiense (Büscher et al., 1999) make use of the native VSGs LiTat 1.3, LiTat 1.5 and/or LiTat 1.6. The sequencing of the VSG LiTat 1.5 gene revealed that the N-terminal domain can be classified as type A even though the third of four typically conserved cysteines is missing. The $\mathrm{C}$-terminal domain is a classical type 2 (Carrington et al., 1991).

\section{MATERIAL AND METHODS}

VSG LiTat 1.5 was purified from a cloned population of $T$. b. gambiense VAT LiTat 1.5 (Büscher et al., 1999). The N-terminal amino acid sequence of the mature VSG LiTat 
1.5 protein was determined by Edman degradation (Edman, 1950) on an ABI 471-B as recommended by the manufacturer. RNA was extracted from the same cloned population as above (RNeasy Midi Kit; Qiagen). After removing contaminating DNA (DNA-free ${ }^{\mathrm{TM}}$ kit; Ambion), cDNA was prepared by RT-PCR with an oligo(dT) $)_{18}$-primer (Transcriptor First-Strand cDNA Synthesis kit; Roche). To amplify the VSG LiTat 1.5 DNA sequence, a proofreading DNA polymerase (Deep Vent ${ }_{R}{ }^{T M}$ DNA polymerase; New England Biolabs) and oligo 54 (spliced leader) forward primer and oligo 42 (VSG all) reverse primer were used (Table 1). The PCR product was purified (QIAquick PCR Purification kit; Qiagen), and post-amplification 3'-adenine ends were added with HotStarTaq ${ }^{\circledR}$ DNA polymerase (Qiagen). The resulting construct was ligated into a $\mathrm{pCR}^{\circledR} 2.1-\mathrm{TOPO}^{\circledR}$ vector (TOPO TA Cloning ${ }^{\circledR}$ Kit; Invitrogen). These plasmids were transformed by heat-shock $\left(30 \mathrm{~s}\right.$ at $\left.42^{\circ} \mathrm{C}\right)$ into One Shot ${ }^{\mathbb{B}}$ TOP10 chemically competent Escherichia coli cells. Clones with VSG LiTat 1.5 inserted in the $\mathrm{pCR}^{\circledR} 2.1-\mathrm{TOPO}^{\circledR}$ vector were screened by colony-PCR with the M13 forward and reverse primers (Table 1). Five positive colonies were grown for plasmid purification with the QIAprep ${ }^{\circledR}$ Spin Miniprep kit (Qiagen). The nucleotide sequence of VSG LiTat 1.5, ligated into the $\mathrm{pCR}^{\circledR} 2.1-\mathrm{TOPO}^{\circledR}$ vector, was determined at the VIB Genetic Service Facility of the University of Antwerp by sequencing with the M13 forward and reverse primers. The chromatograms were read with Chromas 2.33 (Technelysium Pty Ltd.), and five identical DNA sequences were obtained.

Table 1. Primers used for the amplification and sequencing of LiTat $1.5 \mathrm{cDNA}$.

\begin{tabular}{ll}
\hline & Primer sequence (5'-3') \\
\hline Oligo 54 (spliced leader) & GACTAGTTTCTGTACTAT \\
Oligo 42 (VSG all) & CCGGGTACCGTGTTAAAATATATC \\
M13 forward primer & TGTAAAACGACGGCCAGT \\
M13 reverse primer & CAGGAAACAGCTATGACC \\
\hline VSG = variant surface glycopotein
\end{tabular}

$\mathrm{VSG}=$ variant surface glycoprotein.

\section{RESULTS AND DISCUSSION}

The cDNA and protein sequence of VSG LiTat 1.5 are published in the NIH genetic sequence database (GenBank ID: HQ662603). The amino acid sequence of VSG LiTat 1.5 is presented in Figure 1.

Fifty-seven N-terminal amino acids were derived by direct sequencing of the mature VSG LiTat 1.5 protein: AAITDADTGPAQITDVxKEEFYLSELRKELAAGITRRRTQRQGLLxIQxxY RLAADL. The signal sequence was determined by comparison of the amino acids obtained by Edman degradation with the sequence derived from cDNA. The N-terminal domain is nominally taken to be residue 1 to 350 of the mature VSG. Interestingly, the cysteine distribution in both the $\mathrm{N}$ - and C-terminal domains of the mature VSG LiTat 1.5 protein is identical to that of VSG Kinu 1 (GenBank ID: AJ937313.1), even though both proteins share only 33\% of identical amino acids (Clustal W multiple sequence alignment (Thompson et al., 1994) (Figure 1).

VSG Kinu 1, of a T. b. brucei strain originally collected in the Tororo district of Uganda, has been classified as a type A N-terminal domain (Hutchinson et al., 2007; Marcello and Barry, 2007), but when compared to typical type A VSGs such as VSG MITat 1.2, the third of four conserved cysteines is missing (Carrington et al., 1991). However, according to Marcello and Barry (2007), a group 2 subclass and a group 4 of type A also 
lack this conserved cysteine around position 123 (Figure 2). Furthermore, the presence of cysteine at position 147 is similar to the group 1 cysteine pattern (MITat 1.2 residue 145), while the cysteines at positions 17, 65, 214, 250,272, and 306 show an identical cysteine pattern as in group 2 subclass of type A. The extra cysteine residue at position 196 can be found in group 4 of type A. Classification of the protein sequences of VSG LiTat 1.5 and Kinu 1 is thus not straightforward. As six of eight cysteines are similar to the group 2 subclass of type A, they can be classified as type A, but it is also possible that they constitute a separate group or subclass.

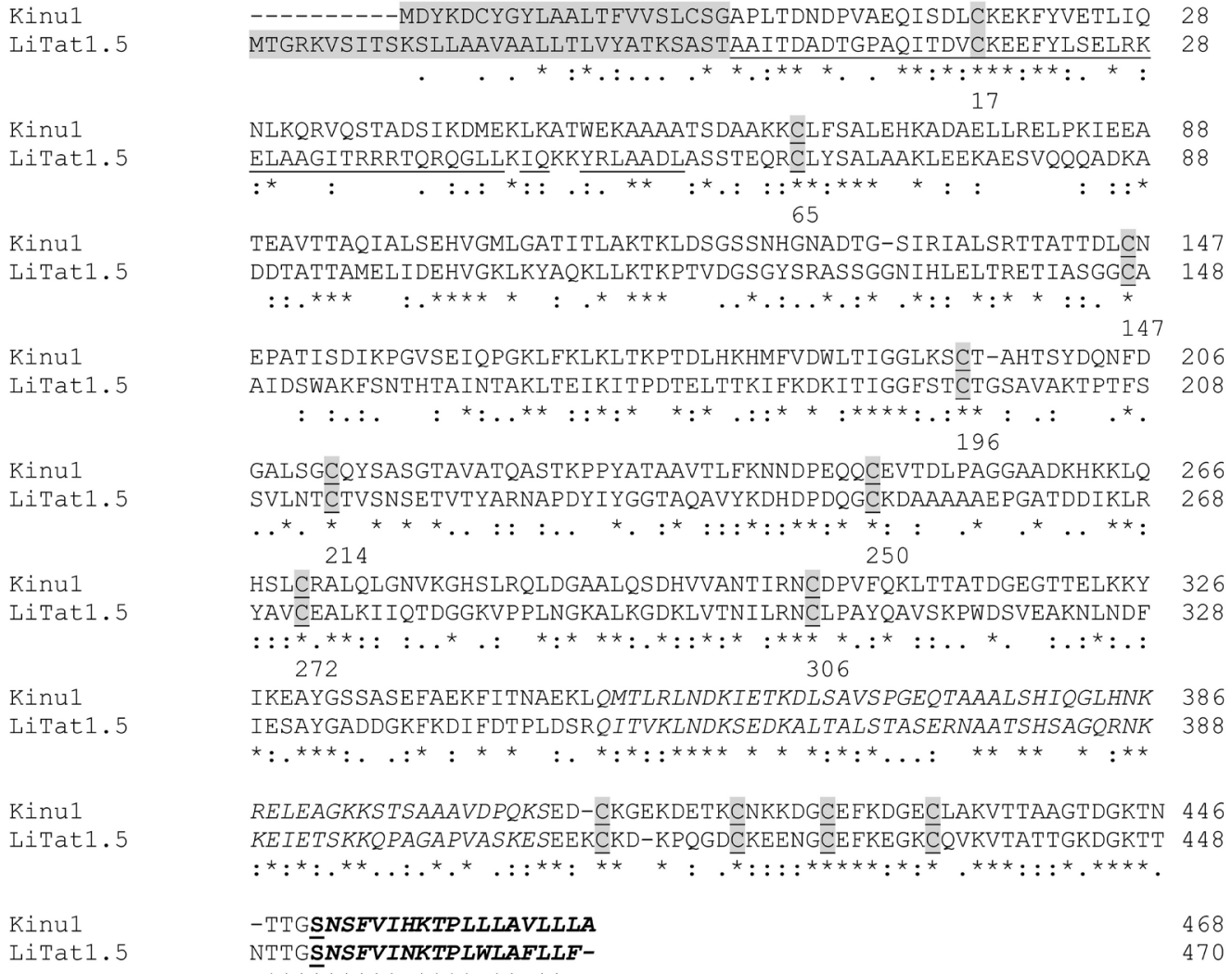

Figure 1. Alignment of variant surface glycoprotein (VSG) LiTat 1.5 of T. $b$. gambiense with VSG Kinu 1 of $T . b$. brucei. The amino acid sequence of LiTat 1.5 was deduced from its cDNA sequence (GenBank ID: HQ662603). The amino acid residues are numbered starting with the $\mathrm{N}$-terminal end of the mature protein as determined by Edman degradation. The result of the Edman degradation is underlined. The amino acid sequence of VSG LiTat 1.5 is aligned with the sequence of Kinu 1 (Genbank ID: AJ937313.1). The signal sequence is indicated in gray. Cysteines are gray and underlined, and their position in the N-terminal domain of the mature protein sequence of VSG LiTat 1.5 is numbered. The domain boundary between the nominally determined mature $\mathrm{N}$ - and C-terminal domains is in italics. The C-terminal hydrophobic sequence is in bold italics. The C-terminal residue is bold and underlined. Identical residues are marked with an asterisk under the sequence. Colons indicate strongly conserved substitutions (side chains with similar biochemical properties, scoring $>0.5$ in the Gonnet PAM 250 matrix). Periods indicate weakly conserved substitutions (scoring $\leq 0.5$ in the Gonnet PAM 250 matrix). 


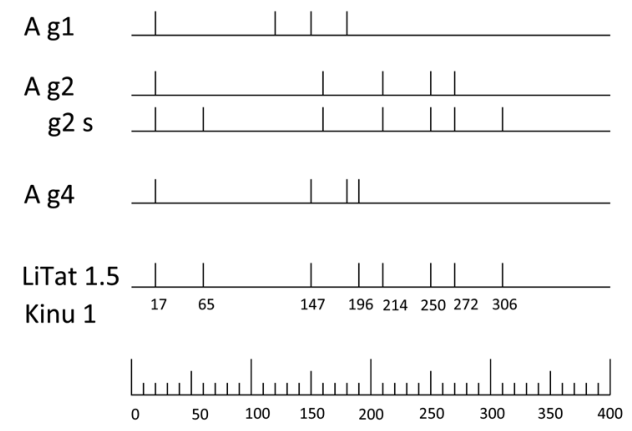

Figure 2. Cysteine pattern of variant surface glycoprotein N-terminal domain types. Cysteine positions are indicated as vertical bars. The scale bar (number of residues) is shown below the figure. $\mathrm{A}=\mathrm{N}$-terminal domain type A; g = group; s = subgroup. Figure adapted from Marcello and Barry (2007).

The location of the mature $\mathrm{C}$-terminal domain and $\mathrm{C}$-terminus is based on sequence homology (Carrington et al., 1991). The C-terminal domain sequence of both VSG LiTat 1.5 and VSG Kinu 1 consists of 45 amino acids and belongs to type 2, with four cysteine residues (positions 412, 420, 426, and 433) and serine as C-terminal residue. The hydrophobic extension of VSG LiTat 1.5 and VSG Kinu 1 contains, respectively, 17 and 18 amino acids, starts with asparagine and shows sequence homology to the hydrophobic extension of VSG MITat 1.2 (Carrington et al., 1991).

In conclusion, the sequence of the predominant VSG LiTat 1.5 of $T$. b. gambiense displays an atypical cysteine pattern in the N-terminal domain, but can nevertheless be classified as a type A. Furthermore, despite a 33\% identical amino acid sequence, VSG LiTat 1.5 shares an identical distribution of cysteine residues, in both the $\mathrm{N}$ - and C-terminal domains, with VSG Kinu 1 of $T$. b. brucei.

\section{ACKNOWLEDGMENTS}

Research supported by the Belgian Directorate General for Development Cooperation (DGDC) and the Research Foundation of Flanders (FWO, \#1.5.169.07.N and \#1.5.074.09.N). L. Van Nieuwenhove was supported by a PhD scholarship from FWO (\#1.1.270.07.N.00 and \#1.1.270.09.N.01). The funders had no role in the study design, data collection and analysis, decision to publish, or preparation of the manuscript. We thank the laboratory of Luc Moens for performing the Edman degradation. We are grateful to Mark Carrington for critical reading of the manuscript.

\section{REFERENCES}

Berriman M, Ghedin E, Hertz-Fowler C, Blandin G, et al. (2005). The genome of the African trypanosome Trypanosoma brucei. Science 309: 416-422.

Blum ML, Down JA, Gurnett AM, Carrington M, et al. (1993). A structural motif in the variant surface glycoproteins of Trypanosoma brucei. Nature 362: 603-609.

Büscher P, Lejon V, Magnus E and Van Meirvenne N (1999). Improved latex agglutination test for detection of antibodies in serum and cerebrospinal fluid of Trypanosoma brucei gambiense infected patients. Acta Trop. 73: 11-20.

Carrington M, Miller N, Blum M, Roditi I, et al. (1991). Variant specific glycoprotein of Trypanosoma brucei consists 
of two domains each having an independently conserved pattern of cysteine residues. J. Mol. Biol. 221: 823-835.

Chattopadhyay A, Jones NG, Nietlispach D, Nielsen PR, et al. (2005). Structure of the C-terminal domain from Trypanosoma brucei variant surface glycoprotein MITat1.2. J. Biol. Chem. 280: 7228-7235.

Edman P (1950). Method for determination of the amino acid sequence in peptides. Acta Chem. Scand. 4: 283-293.

Engstler M, Thilo L, Weise F, Grunfelder CG, et al. (2004). Kinetics of endocytosis and recycling of the GPI-anchored variant surface glycoprotein in Trypanosoma brucei.J. Cell Sci. 117: 1105-1115.

Engstler M, Pfohl T, Herminghaus S, Boshart M, et al. (2007). Hydrodynamic flow-mediated protein sorting on the cell surface of trypanosomes. Cell 131: 505-515.

Ferguson MA, Homans SW, Dwek RA and Rademacher TW (1988a). Glycosyl-phosphatidylinositol moiety that anchors Trypanosoma brucei variant surface glycoprotein to the membrane. Science 239: 753-759.

Ferguson MAJ, Homans SW, Dwek RA and Rademacher TW (1988b). The glycosylphosphatidylinositol membrane anchor of Trypanosoma brucei variant surface glycoprotein. Biochem. Soc. Trans. 16: 265-268.

Freymann DM, Metcalf P, Turner M and Wiley DC (1984). 6 A-resolution X-ray structure of a variable surface glycoprotein from Trypanosoma brucei. Nature 311: 167-169.

Hutchinson OC, Smith W, Jones NG, Chattopadhyay A, et al. (2003). VSG structure: similar N-terminal domains can form functional VSGs with different types of C-terminal domain. Mol. Biochem. Parasitol. 130: 127-131.

Hutchinson OC, Picozzi K, Jones NG, Mott H, et al. (2007). Variant Surface Glycoprotein gene repertoires in Trypanosoma brucei have diverged to become strain-specific. BMC Genomics 8: 234.

Magnus E, Vervoort T and Van Meirvenne N (1978). A card-agglutination test with stained trypanosomes (C.A.T.T.) for the serological diagnosis of T. B. gambiense trypanosomiasis. Ann. Soc. Belg. Med. Trop. 58: 169-176.

Marcello L and Barry JD (2007). Analysis of the VSG gene silent archive in Trypanosoma brucei reveals that mosaic gene expression is prominent in antigenic variation and is favored by archive substructure. Genome Res. 17: 1344-1352.

Schwede A and Carrington M (2010). Bloodstream form Trypanosome plasma membrane proteins: antigenic variation and invariant antigens. Parasitology 137: 2029-2039.

Schwede A, Jones N, Engstler M and Carrington M (2011). The VSG C-terminal domain is inaccessible to antibodies on live trypanosomes. Mol. Biochem. Parasitol. 175: 201-204.

Thompson JD, Higgins DG and Gibson TJ (1994). CLUSTAL W: improving the sensitivity of progressive multiple sequence alignment through sequence weighting, position-specific gap penalties and weight matrix choice. Nucleic Acids Res. 22: 4673-4680.

Van Meirvenne N, Magnus E and Buscher P (1995). Evaluation of variant specific trypanolysis tests for serodiagnosis of human infections with Trypanosoma brucei gambiense. Acta Trop. 60: 189-199. 